\title{
Andreas GOCKSCH
}

Physics Department, Brookhaven National Laboratory, Upton, New York, 11973

I discuss some recent work on the Hybrid Monte Carlo algorithm as applied to the simulation of dynamical fermions as well as some interesting aspects of the nature of the phase transition at $\kappa_{\mathrm{c}}$ in the Wilson fermion formulation.

At this meeting I reviewed some recent results that were presented at the lattice gauge theory workshop which took place at the University of Kentucky from July 27 th to August 4th 1989 . In this written version of the talk I will - because of lack of space- concentrate on orly one topic. Actually it's two topics somewhat deceivingly covered under the same title because both involve fermions on the lattice. First I will talk about some new developments in Hybrid Monte Carlo; this is material that was covered by M. Creutz, R. Gupta and A. Kennedy at Lexington. Then I will discuss some work on the phase structure of Wilson fermions that I did in collaboration with S. Aoki.

The Hybrid Monte Carlo Algorithm ${ }^{1}$ seems to have emerged as the algorithm of choice for the simulation of systems with many fermions. Let us quickly recall how it works. We want to generate a probability distribution

$$
P_{e q}(A)=e^{-S(A)}
$$

for a bosonic field $A(x)$ described by an action $S$. (the fermions have been integrated out.) Consider the classical Hamiltonian

$$
H(p, A)=\frac{p^{2}}{2}+S(A)
$$

and a discretization $T_{\delta}:(A, p) \mapsto\left(\hat{A}^{\prime}, p^{\prime}\right)$ of Hamilton's equations. The momentum $p$ is a gaussian random variable of unit width and $\delta$ represents the step size. Then it is not hard to show that as long as $F_{\delta}$ is both reversible $\left(T^{-1}=T_{-\delta}\right)$ and area preserving $\left((d A, d p)=\left(d A^{\prime}, d p^{\prime}\right)\right)$, simply accepting a step of the entire lattice under $T$ with probability

$$
P_{\text {ucc }}=\min \left[1, e^{H(p, A)-H\left(p^{\prime}, A^{\prime}\right)}\right]
$$

will exactly satisfy detailed balance and hence result in the desired probability distribution. After each accept/reject the momenta are refreshed. Clearly, since the algorithm is a global one, the change in energy (3) will be multiplied by a factor of the volume resulting in a possibly very small acceptance rate. Hence it is desirable to follow a classical trajectory as closely as possible for if we were to follow it txaclly the acceptance would be unity. A discretization of Newtons equations which has an error starting at $\delta^{3}$ is the so called leapfrog method. It involves taking a half-step in (say) $p$ then a step in $A$ and finally another half step in $p$. It can be shown ${ }^{2,3}$ that with the leapfrog method the slowing down of the algorithm as a function of the volume at fixed gauge coupling is proportional to $V^{\frac{5}{4}}$.

This result has recently been extended ${ }^{4}$ to also include the effect of the critical slowing down at small quark masses. The result is that the computer time grows as $\left(\frac{1}{m}\right)^{\frac{13}{4}}$ - a somewhat discouraging prospect. Nevertheless as long as one does not make the quark mass too small the algorithm performs well. In their detailed study of the algorithm using Wilson fermions Gupta et. al. ${ }^{4}$ conclude that even on a $8^{4}$ lattice one can achieve a $60-70$ per cent acceptance rate without having to make $\delta$ smaller than that used in the algorithm without the final accept/reject.

The asymptotic volume dependence of the al- 
gorithm can be improved by using a higher order integration scheme. A scheme which is extremely easy to implement and which gives the equations of motion accurate to any desired order while at the same time being area preserving and reversible (this is of course of interest not only to lattice gauge theorists) was recently discovered ${ }^{5}$. It works as follows: Assume a transformation $T_{n}(\delta)$ is a time reversal invariant, area preserving discretization of Hamilton's equations to order $n=2 m$, i.e. the error is of order $\delta^{2 m+1}$. Then for an arbitrary integer $i$ one takes $i$ steps of size $\delta$, then a step backward of size $s \delta=(2 i)^{\frac{1}{n+1}} \delta$ and finally makes $i$ more steps forward. Then by construction

$$
T_{n+2}((2 i-s) \delta)=T_{n}(\delta)^{i} T_{n}(-s \delta) T_{n}(\delta)^{i}
$$

is accurate to order $n+2$. For example, to get an algorithm accurate to order $\delta^{5}$ we call the leapfrog subroutine three times and use $s=2^{\frac{1}{3}}$. The asymptotic volume dependence of the algorithm can be shown to be $V e^{\text {const }} \sqrt{\log V}$ which has a correction to linear behavior slower than any power. This is an asymptotic statement however - in tests of the method in the Hubbard model the standard leapfrog discretization performed best on the lattices used. It would be interesting to check where 'asymptopia' begins in QCD.

An interesting observation concerning ergodicity in the Hybrid Monte Carlo algorithm was recently made by Mackenzie ${ }^{6}$. Consider free field theory. Then under the microcanonical evolution a particular mode of the system (Fourier component of $\phi$ ) evolves as

$$
\dot{\phi}_{p}(\tau)=\phi_{p}(0) \cos \left(\omega_{p} \tau\right)+\frac{\pi_{p}(0)}{\omega_{p}} \sin \left(\omega_{p} \tau\right)
$$

where $\pi_{p}$ is the conjugate momentum of $\phi$. Hence if the trajectory length $\tau$ is close to 'resonance', i.e. $\left|\frac{2 n \pi}{\omega_{p}}-T\right|<\epsilon$, $\phi$ only evolves by a amount $\epsilon$. Mackenzie showed that given a trajectory length $\tau$ greater than the time scale set by the fastest mode in the system $T_{\min }=\frac{2 \pi}{\omega_{\max }}$ one is actually quite likely to find some modes that satisfy the above inequality. This is the reason why simply choosing $\tau$ of the order of $T_{\max }$ does not. randomize the system after one (or a few) trajectories. There are always some
- modes that need $O\left(\frac{1}{\epsilon^{2}}\right)$ trajectories to rañdortí walk a distance one. The problem persists in an interacting system. The solution to this problem with ergodicity of some modes is to randomize the trajectory length during the simulation. This way on each trajectory different modes evolve only by a small amount.

Let me now come to the second subject that 1 want to cover here, namely the nature of the phase transition at $\kappa_{c}$ in the Wilson formulation of lattice fermions. The standard lore on the subject is of course that at $\kappa_{c}$ the quarks become massless, chiral symmetry is realized in the spontaneously broken mode and the pion is a goldstone boson. This however is a staioment about the continuum limit. If the lattice spacing $a$ is finite there are $O(a)$ corrections to any relation derived from the assumption of spontaneously broken chiral symmetry. This follows most readily from the investigation of current algebra relations using Wilson fermions ${ }^{7}$. One has to wonder then why the pion comes out to be a massless particle at $\kappa_{c}$ even at a finite lattice spacingan extreme example being the case of infinite gauge coupling. Aoki ${ }^{8}$ suggested the following scenario: At $\kappa_{c}$ there is an ordinary second order phase transition with order parameter $\bar{\psi} i \gamma_{5} \psi$, i.e. beyond that point one is in a phase with spontaneously broken parity. The pion is to be identified with the inverse of the diverging correlation length at the critical point and is therefore (at a finite lattice spacing) not to be identified with a goldstone boson. The syrrmetry that is broken here is discrete. But note tha: the famous relation $m_{\pi}{ }^{2} \sim m_{q}$ still holds even at finite $a$. It is just a consequence of the mean field nature of the phase transition with a critical exponent $\nu$ of one half. Recently Aoki and myself ${ }^{9}$ have established the existence of the parity violating phase in the quenched approximation. Using a parity breaking term $H \vec{\psi} i \gamma_{5} \psi$ in the action we showed that on a $4^{4}$ lattice at $\beta=5.0$ beyond $\kappa_{c}=0.205(5)$ a finite parity breaking condensate remains in the limit $H \mapsto 0$. Since then we have extended our work to the case of finite temperature $Q C D$. The result ${ }^{10}$ is that paiity is broken beyond some $\kappa_{c}$ in the confined phase and is always a rood symmetry in the deconfined phase (Our simulations were done on $\delta^{3} \cdot 4$ lattice at $\beta=5.6$ and 5.8). The last fact is important 
Figure 1: The screening masses as a function of $k$ at $\beta=5.865$ on a $8^{2} \cdot 20 \cdot 4$ lattice. The data points represent $\pi$ (solid circle), $\sigma$ (open circle), $\rho$ (solid triangle), $A_{1}$ (open triangle), $N_{+}$(solid square) and $N_{-}$(open square).

because it can be used to look at the amount of explicit chiral symmetry breaking due to the finiteness of the lattice spacing in the high temperature phase. If chiral symmetry were compietely restored at high temperatures we would expect hadronic modes to be parity doubled. As can be seen from the figure there seems however to always remain a finite splitting no matter what $\kappa$ is, in particular around $\kappa \sim 0.16$ which is where the 'critical kappa' should be located according to the ward identity definition used by lwasaki et. al. 11 . This should be contrasted with an identical (quenched) simulation using KogutSusskind fermions ${ }^{12}$ where one gets complete parity doubling in the limit $m_{q} \mapsto 0$. We interpret this fact as an indication of the remaining breaking of chiral symmetry due to the finite lattice spacing in the Wilson formulation.
What does all this mean? First of all the existence of spontaneous breaking of parity is by itself an interesting phenomenon. Furthermore it means thar we must be careful when talking about chiral symmetry at $\kappa_{\mathrm{c}}$ using Wilson fermions. In particular the goldstone boson nature of the pion is strictly speaking only a property of the continuum limit and the the above relation between pion and quark masses should not be taken as an indication for the 'good chiral behavior' of Wilson fermions. This does of course not mean that Wilson fermions do not have the right chiral properties in the continuum limit. As a matter of fact they do as the analysis of ref. 7 shows.

\section{ACKNOWLEDGEMENTS}

I would like to thank A. Gonzalez Arroyo for his efforts in organizing this session. This manuscript has been authored under Contract No. DE-AC02$76 \mathrm{CH} 00016$ with the U.S. Department of Energy.

\section{REFERENCES}

1. S. Duane, A.D. Kennedy, B.J. Pendleton and D. Roweth, Phys. Lett. $195 B$ (1987) 216

2. M. Creutz, Phys. Rev. D38 (1988) 1228

3. R. Gupta, G. Kilcup and S. Sharp, Phys. Rev. D38 (1988) 1278

4. R. Gupta, G. Kilcup and S. Sharp, Phys. Rev. D40 (1989) 2072

5. M. Creutz and A. Gocksch, Phys. Rev. Lett. 63 (1989) 9

6. Phys. Lett. 226B (1989) 369

7. M. Bochicchio et. al., Nucl. Phys. B262 (1985) 331

8. S. Aoki, Phys. Rev. Lett. 57 (1986) 3136; Nud. Phys. B314 (1986) 79

9. S. Aoki and A. Gocksch, preprint BNL-43054 (1989), to be published in Phys. Lett. B.

10. S. Aoki and A. Gocksch, in preparation

11. Y. Iwasaki, Y. Tsuboi and T. Yoshie, Phys. Lett. 220B (1989) 602

12. A. Gocksch, U.Heller and P.Rossi, Phys. Lett. 205B (1988) 334 